\title{
ASYMPTOTIC JOINT SPECTRA OF CARTESIAN POWERS OF STRONGLY REGULAR GRAPHS AND BIVARIATE CHARLIER-HERMITE POLYNOMIALS
}

\author{
JOHN VINCENT S. MORALES, NOBUAKI OBATA, AND HAJIME TANAKA
}

\begin{abstract}
Generalizing previous work of Hora (1998) on the asymptotic spectral analysis for the Hamming graph $H(n, q)$ which is the $n^{\text {th }}$ Cartesian power $K_{q}^{\square n}$ of the complete graph $K_{q}$ on $q$ vertices, we describe the possible limits of the joint spectral distribution of the pair $\left(G^{\square n}, \bar{G}^{\square n}\right)$ of the $n^{\text {th }}$ Cartesian powers of a strongly regular graph $G$ and its complement $\bar{G}$, where we let $n \rightarrow \infty$, and $G$ may vary with $n$. This result is an analogue of the bivariate central limit theorem, and we obtain in this way the bivariate Poisson distributions and the standard bivariate Gaussian distribution, together with the product measures of univariate Poisson and Gaussian distributions. We also report a family of bivariate hypergeometric orthogonal polynomials with respect to the last distributions, which we call the bivariate Charlier-Hermite polynomials, and prove basic formulas for them. This family of orthogonal polynomials seems previously unnoticed, possibly because of its peculiarity.
\end{abstract}

\section{INTRODUCTION}

Let $G=(V, E)$ be a finite simple graph with vertex set $V$ and edge set $E$. (Formal definitions about graphs will be given in Section 2.) The adjacency matrix $A$ of $G$ is the 0-1 matrix indexed by $V$, where $A_{x, y}=1$ if and only if $x$ and $y$ are adjacent. By an eigenvalue of $G$ we mean an eigenvalue of $A$. Likewise, we speak of the spectrum of $G$.

Spectra of graphs have been receiving attention from the point of view of quantum probability theory. Recall that an algebraic probability space is a pair $(\mathcal{A}, \varphi)$, where $\mathcal{A}$ is a $*$-algebra over $\mathbb{C}$ and $\varphi: \mathcal{A} \rightarrow \mathbb{C}$ is a state, i.e., a linear map such that $\varphi(1)=1$ and that $\varphi\left(a^{*} a\right) \geqslant 0$ for every $a \in \mathcal{A}$. The elements of $\mathcal{A}$ are referred to as (algebraic) random variables. We call $a \in \mathcal{A}$ real if $a^{*}=a$. For a real random variable $a \in \mathcal{A}$, we are interested in finding, and discussing the uniqueness of, a probability measure $\nu$ on $\mathbb{R}$ such that

$$
\varphi\left(a^{j}\right)=\int_{-\infty}^{+\infty} x^{j} \nu(d x) \quad(j=0,1,2, \ldots) .
$$

Associated with the graph $G$ above is the adjacency algebra $\mathbb{C}[A]$, i.e., the commutative subalgebra of the full matrix algebra generated by $A$. For the $*$-algebra

2010 Mathematics Subject Classification. Primary 46L53; Secondary 60F05, 05E30, 33C45.

Key words and phrases. Quantum probability, central limit theorem, spectral distribution, strongly regular graph, orthogonal polynomial, hypergeometric series. 
$\mathbb{C}[A]$, it is natural to consider the tracial state $\varphi_{\text {tr }}$ defined by 1$]$

$$
\varphi_{\text {tr }}(X)=\frac{1}{|V|} \operatorname{tr}(X) \quad(X \in \mathbb{C}[A]) .
$$

Suppose for simplicity that $G$ is $k$-regular, so that $A$ has mean $\varphi_{\operatorname{tr}}(A)=0$ and variance $\varphi_{\operatorname{tr}}\left(A^{2}\right)=k$. Let $k=\theta_{0}>\theta_{1}>\cdots>\theta_{d}$ be the distinct eigenvalues of $G$, and let $m_{j}$ be the multiplicity of $\theta_{j}$. Then the unique probability measure $\nu=\nu_{G}$ in (11) for $a=A / \sqrt{k}$ is the (normalized) spectral distribution of $G$ given by

$$
\nu_{G}\left(\frac{\theta_{j}}{\sqrt{k}}\right)=\frac{m_{j}}{|V|} \quad(j=0,1, \ldots, d) .
$$

Our focus is on the limit of $\nu_{G}$ when $G$ "grows", as an analogue of the classical central limit theorem. Hora [14 described various limit distributions for several growing families of Cayley graphs and distance-regular graphs (cf. [4]). For example, for the Hamming graphs $H(n, q)$ which are one of the most important families of distance-regular graphs, he obtained a Poisson distribution when $q / n \rightarrow \tau(n \rightarrow \infty)$ where $0<\tau<\infty$, and the standard Gaussian distribution when $q / n \rightarrow 0(n \rightarrow \infty)$. Hora worked with the spectra directly in [14, but then Hora, Obata, and others revisited, simplified, and generalized these results based on the idea of decomposing the random variable $A$ into the sum of certain three non-commuting components $A^{+}, A^{\circ}, A^{-}$in a larger $*$-algebra 3 called the quantum decomposition of $A$. Besides the Poisson and Gaussian distributions, many important univariate distributions arise in this way, such as the exponential, geometric, gamma, and the two-sided Rayleigh distributions. See, e.g., [11, 12, 15, 16, for more details.

The purpose of the present paper is to give a concrete bivariate example of this sort, as an attempt towards a multivariate extension of the theory. Consider again a general algebraic probability space $(\mathcal{A}, \varphi)$. We now pick two commuting real random variables $a, b \in \mathcal{A}$, and discuss a probability measure $\nu$ on $\mathbb{R}^{2}$ such that

$$
\varphi\left(a^{j} b^{h}\right)=\int_{\mathbb{R}^{2}} x^{j} y^{h} \nu(d x d y) \quad(j, h=0,1,2, \ldots) .
$$

In our context, we take another (say, $\ell$-regular) graph $H=(V, F)$ having the same vertex set $V$ as $G$, and assume that the adjacency matrix $B$ of $H$ commutes with $A$. This occurs for example when $H=\bar{G}$, the complement of $G$. (Recall that we are assuming that $G$ is $k$-regular.) We view $A$ and $B$ as real random variables in the algebraic probability space $\left(\mathbb{C}[A, B], \varphi_{\operatorname{tr}}\right)$. Note that the covariance $\varphi_{\operatorname{tr}}(A B)$ for $A$ and $B$ equals 0 if and only if $G$ and $H$ have no edge in common, i.e., $E \cap F=\emptyset$. Let $\ell=\eta_{0}>\eta_{1}>\cdots>\eta_{e}$ be the distinct eigenvalues of $H$, and let $m_{j, h}$ be the dimension of the common eigenspace of $(A, B)$ with respective eigenvalues $\left(\theta_{j}, \eta_{h}\right)$. The probability measure $\nu=\nu_{G, H}$ in (2) for $a=A / \sqrt{k}$ and $b=B / \sqrt{\ell}$ is then the (normalized) joint spectral distribution of $G$ and $H$ given by

$$
\nu_{G, H}\left(\frac{\theta_{j}}{\sqrt{k}}, \frac{\eta_{h}}{\sqrt{\ell}}\right)=\frac{m_{j, h}}{|V|} \quad(j=0,1, \ldots, d, h=0,1, \ldots, e) .
$$

\footnotetext{
${ }^{1}$ Another important example is the vacuum state $\varphi_{x}(X)=X_{x, x}(X \in \mathbb{C}[A])$ at a fixed origin $x \in V$. We note that the matrix $*$-algebras we will discuss in this paper all have the property that every element has constant diagonal entries, so that the two states $\varphi_{\operatorname{tr}}$ and $\varphi_{x}$ turn out to be equal on them.

${ }^{2}$ We may remark that $A^{+}, A^{\circ}$, and $A^{-}$belong to the Terwilliger algebra 22 of $G$. See [4, Section 16.6].
} 
We are again interested in the limit of $\nu_{G, H}$ when $G$ and $H$ both grow, as an analogue of the bivariate central limit theorem.

Our main result (Theorem 2.1) is indeed a bivariate version of the result of Hora 14 for the Hamming graphs mentioned above. The Hamming graph $H(n, q)$ is defined as the $n^{\text {th }}$ Cartesian power $K_{q}^{\square n}$ of the complete graph $K_{q}$ on $q$ vertices. We will instead consider the pair $\left(G^{\square n}, \bar{G}^{\square n}\right)$ of the $n^{\text {th }}$ Cartesian powers of a strongly regular graph $G$ and its complement $\bar{G}$, and obtain as limits the bivariate Poisson distributions and the standard bivariate Gaussian distribution, together with the product measures of univariate Poisson and Gaussian distributions. The method of quantum decomposition is yet to be developed for the multivariate case, and hence we will deal with the spectra of these graphs directly, as was done by Hora in [14, though the discussions here become much more involved. We note that the complete graphs are the connected regular graphs with precisely two distinct eigenvalues, whereas the connected strongly regular graphs are those with precisely three distinct eigenvalues. This comparison can be made clearer when viewed in the framework of association schemes, and our choice of considering the pair $\left(G^{\square n}, \bar{G}^{\square n}\right)$ above was in fact guided naturally by the work of Mizukawa and Tanaka [18] on a construction of multivariate Krawtchouk polynomials from arbitrary association schemes. See Section 6. As a by-product, we report in Section 6 a family of bivariate hypergeometric orthogonal polynomials with respect to the last distributions, which we call the bivariate Charlier-Hermite polynomials, and prove basic formulas for them. This family of orthogonal polynomials seems previously unnoticed, possibly because of its peculiarity.

Section 3 collects necessary facts about strongly regular graphs. Section 4 is devoted to the proof of Theorem 2.1. In Section [5, we demonstrate Theorem 2.1 with some specific families of strongly regular graphs.

\section{BASIC DEFINITIONS AND THE MAIN RESUlT}

Let $G=(V, E)$ be a graph with vertex set $V$ and edge set $E$. All the graphs we consider in this paper are finite and simple. Thus, $V$ is a finite set and $E$ is a subset of $\left(\begin{array}{c}V \\ 2\end{array}\right)$, the set of 2-element subsets of $V$. The elements of $V$ are vertices of $G$ and the elements of $E$ are edges of $G$. Two vertices $x, y \in V$ are called adjacent (and written $x \sim y$ ) if $\{x, y\} \in E$. The degree (or valency) $k(x)$ of a vertex $x \in V$ is the number of vertices adjacent to $x$. The graph $G$ is called $k$-regular if $k(x)=k$ for all $x \in V$. It is called connected if for any two vertices $x$ and $y$, there is a sequence of vertices $x=x_{0}, x_{1}, \ldots, x_{t}=y$ such that $x_{j-1} \sim x_{j}$ for $j=1,2, \ldots, t$. Recall that a complete graph $K_{v}$ is a graph on $|V|=v$ vertices such that $E=\left(\begin{array}{c}V \\ 2\end{array}\right)$. As in Introduction, the adjacency matrix $A$ of $G$ is the matrix indexed by $V$ such that $A_{x, y}=1$ if $x \sim y$ and $A_{x, y}=0$ otherwise. The complement $\bar{G}$ of $G$ is the graph with the same vertex set $V$ as $G$, where two distinct vertices are adjacent if and only if they are non-adjacent in $G$. Thus, $\bar{G}$ has adjacency matrix $\bar{A}:=J-A-I$, where $I$ and $J$ denote the identity matrix and the all-ones matrix, respectively.

The Cartesian product $G_{1} \square G_{2}$ of two graphs $G_{j}=\left(V_{j}, E_{j}\right)(j=1,2)$ is the graph with vertex set $V_{1} \times V_{2}$, where $\left(x_{1}, x_{2}\right) \sim\left(y_{1}, y_{2}\right)$ if and only if either $x_{1} \sim y_{1}$ and $x_{2}=y_{2}$, or $x_{1}=y_{1}$ and $x_{2} \sim y_{2}$; cf. [3, Section 1.4.6]. For a positive integer $n$, the Cartesian power $G \square G \square \cdots \square G$ ( $n$ times) will be denoted by $G^{\square n}$. For example, we already mentioned that $H(n, q)=K_{q}^{\square n}$. The adjacency matrix $\boldsymbol{A}$ of 
$G^{\square n}$ is given by

$$
\boldsymbol{A}=\sum_{j=1}^{n} I \otimes \cdots \otimes I \otimes \underset{j}{A} \otimes I \otimes \cdots \otimes I .
$$

From now on, suppose that $G$ is $k$-regular and has $|V|=v$ vertices. We note that $k$ is an eigenvalue of $G$ (i.e., of $A$ ), and that every eigenvalue $\theta$ of $G$ satisfies $|\theta| \leqslant k$; cf. [3. Section 1.3.1]. We have $A J=J A=k J$, and hence $A \bar{A}=\bar{A} A$. Observe that $G^{\square n}$ is $n k$-regular, and that $\boldsymbol{A} \overline{\boldsymbol{A}}=\overline{\boldsymbol{A}} \boldsymbol{A}$, where $\overline{\boldsymbol{A}}$ denotes the adjacency matrix of $\bar{G}^{\square n}$. Since $G^{\square n}$ and $\bar{G}^{\square n}$ have no edge in common, the covariance $\varphi_{\operatorname{tr}}(\boldsymbol{A} \bar{A})=0$.

We call $G$ strongly regular with parameters $(v, k, \lambda, \mu)$ if $G$ is not complete or edgeless (i.e., $0<k<v-1$ ), and if every pair of adjacent (resp. non-adjacent and distinct) vertices has precisely $\lambda$ (resp. $\mu$ ) common adjacent vertices; cf. [3, Section 9.1]. In matrix terms, this means that

$$
A^{2}=k I+\lambda A+\mu \bar{A} \text {. }
$$

It is clear that $G$ is a disconnected strongly regular graph precisely when it is the disjoint union $p K_{q}$ of $p$ complete graphs $K_{q}$ for some integers $p, q \geqslant 2$. It is easy to see that if $G$ is strongly regular as above then $\bar{G}$ is again strongly regular with parameters $(v, \bar{k}, \bar{\lambda}, \bar{\mu})$, where

$$
\bar{k}=v-k-1, \quad \bar{\lambda}=v-2 k+\mu-2, \quad \bar{\mu}=v-2 k+\lambda .
$$

Thus, strongly regular graphs always exist in pairs. The complement of $p K_{q}$ is the complete multipartite graph $K_{p \times q}$.

Observe that $G$ is complete if and only if the linear span $\langle I, A\rangle$ equals $\langle I, J\rangle$, which is a two-dimensional *-algebra. Likewise, from (4) it follows that $G$ is strongly regular as above if and only if $\langle I, A, \bar{A}\rangle=\langle I, A, J\rangle$ is a three-dimensional (commutative) *-algebra. Suppose now that this is the case. Then it follows that there are exactly three (maximal) common eigenspaces for $(A, \bar{A})$, one of which corresponds to the eigenvalues $(k, \bar{k})$ and is spanned by the all-ones vector $\mathbf{1}$ in $\mathbb{C}^{v}$. Let $(r, \bar{s})$ and $(s, \bar{r})$ denote the eigenvalues corresponding to the other two, where we have $\bar{s}=-r-1$ and $\bar{r}=-s-1$. We will assume that $r>s$, or equivalently, $\bar{r}>\bar{s}$. We have $s, \bar{s}<0$ since $\operatorname{tr}(A)=\operatorname{tr}(\bar{A})=0$, so that ${ }^{3}$

$$
-1<r \leqslant k, \quad-k \leqslant s<0, \quad-1<\bar{r} \leqslant \bar{k}, \quad-\bar{k} \leqslant \bar{s}<0 .
$$

We call $r$ and $s$ (resp. $\bar{r}$ and $\bar{s}$ ) the restricted 1 eigenvalues of the strongly regular graph $G$ (resp. $\bar{G}$ ).

Notation \& Assumption. We consider an infinite family of pairs of Cartesian powers of graphs $\left(G^{\square n}, \bar{G}^{\square n}\right)$, where $n$ ranges over an infinite set of positive integers, and $G$ is strongly regular and may vary with $n$. To simplify notation, we think of $G, v, k, \bar{k}, r, s$, etc., as functions in $n$. We will assume that

$$
\frac{k}{n} \rightarrow \kappa, \quad \frac{\bar{k}}{n} \rightarrow \bar{\kappa}, \quad \frac{r}{n} \rightarrow \rho, \quad \frac{s}{n} \rightarrow \sigma
$$

as $n \rightarrow \infty$, where $\kappa, \bar{\kappa}, \rho$, and $\sigma$ are finite. We note that

$$
\frac{v}{n} \rightarrow \omega:=\kappa+\bar{\kappa} \text {. }
$$

\footnotetext{
${ }^{3}$ In fact, it follows that $r, \bar{r} \geqslant 0$ and $s, \bar{s} \leqslant-1$; cf. Lemma 3.1

${ }^{4}$ More generally, an eigenvalue of a (not necessarily regular) graph is called restricted if it has an eigenvector which is not a scalar multiple of $\mathbf{1}$.
} 
The following is our main result which describes the possible limits of the joint spectral distribution $\nu_{G}^{\square n}, \bar{G}^{\square n}$.

Theorem 2.1. With the above notation and assumption, we have $\rho=0$ or $\sigma=0$, and one of the following holds:

(i) $\kappa>0, \bar{\kappa}=-\sigma>0, \rho=0$, and $\nu_{G} \square_{n}, \bar{G} \square n$ converges weakly to an affine transformation $\nu$ of a bivariate Poisson distribution given by

$$
\nu\left(\frac{\kappa j-\bar{\kappa} h}{\sqrt{\kappa}}, \frac{\bar{\kappa} j+\bar{\kappa} h-1}{\sqrt{\bar{\kappa}}}\right)=e^{-1 / \bar{\kappa}}\left(\frac{1}{\omega}\right)^{j}\left(\frac{\kappa}{\omega \bar{\kappa}}\right)^{h} \frac{1}{j ! h !}
$$

for $j, h=0,1,2, \ldots$. In this case, $G$ is a complete multipartite graph for all but finitely many values of $n$.

(ii) $\kappa=\rho>0, \bar{\kappa}>0, \sigma=0$, and $\nu_{G} \square_{n}, \bar{G} \square n$ converges weakly to an affine transformation $\nu$ of a bivariate Poisson distribution given by

$$
\nu\left(\frac{\kappa j+\kappa h-1}{\sqrt{\kappa}}, \frac{\bar{\kappa} j-\kappa h}{\sqrt{\bar{\kappa}}}\right)=e^{-1 / \kappa}\left(\frac{1}{\omega}\right)^{j}\left(\frac{\bar{\kappa}}{\omega \kappa}\right)^{h} \frac{1}{j ! h !}
$$

for $j, h=0,1,2, \ldots$ In this case, $G$ is a disjoint union of complete graphs for all but finitely many values of $n$.

(iii) $\kappa>0$ or $\bar{\kappa}>0$, and $\rho=\sigma=0$, and $\nu_{G \square n}, \bar{G} \square n$ converges weakly to an affine transformation $\nu$ of the product measure of a Poisson distribution and a Gaussian distribution given by

$$
\int_{\mathbb{R}^{2}} \gamma(x, y) \nu(d x d y)=\sqrt{\frac{\omega}{2 \pi}} e^{-1 / \omega} \sum_{h=0}^{\infty}\left(\frac{1}{\omega}\right)^{h} \frac{1}{h !} \int_{-\infty}^{+\infty} \gamma\left(\boldsymbol{z}_{h, t}\right) e^{-\omega t^{2} / 2} d t
$$

for every Borel function $\gamma: \mathbb{R}^{2} \rightarrow \mathbb{R}$, where

$$
\boldsymbol{z}_{h, t}=\left(\sqrt{\kappa} h+\sqrt{\bar{\kappa}} t-\frac{\sqrt{\kappa}}{\omega}, \sqrt{\bar{\kappa}} h-\sqrt{\kappa} t-\frac{\sqrt{\bar{\kappa}}}{\omega}\right) .
$$

(iv) $\kappa=\bar{\kappa}=\rho=\sigma=0$, and $\nu_{G} \square_{n}, \bar{G} \square n$ converges weakly to the standard bivariate Gaussian distribution.

\section{Preliminaries on Strongly Regular GraphS}

In this section, we collect necessary facts about strongly regular graphs. See 3 , Chapter 9] for more details. Throughout this section, let $G$ be a (fixed) strongly regular graph with parameters $(v, k, \lambda, \mu)$, and let $\bar{G}$ be the complement of $G$, having parameters $(v, \bar{k}, \bar{\lambda}, \bar{\mu})$ (cf. (5)). Let $A$ (resp. $\bar{A}$ ) be the adjacency matrix of $G$ (resp. $\bar{G})$. For convenience, we let

$$
\mathfrak{k}=(k, \bar{k}), \quad \mathfrak{r}=(r, \bar{s}), \quad \mathfrak{s}=(s, \bar{r}) .
$$

Let $\mathbb{U}_{\mathfrak{k}}, \mathbb{U}_{\mathfrak{r}}$, and $\mathbb{U}_{\mathfrak{s}}$ be the common eigenspaces of $(A, \bar{A})$ associated with $\mathfrak{k}, \mathfrak{r}$, and $\mathfrak{s}$, respectively. Recall that $\mathbb{U}_{\mathfrak{k}}=\langle\mathbf{1}\rangle$. Let

$$
f=\operatorname{dim}\left(\mathbb{U}_{\mathfrak{r}}\right), \quad g=\operatorname{dim}\left(\mathbb{U}_{\mathfrak{s}}\right) .
$$


There are a number of standard identities involving these scalars. What we will need in the proof of Theorem 2.1 are the following:

$$
\begin{gathered}
v=1+k+\bar{k}=1+f+g, \\
0=1+r+\bar{s}=1+s+\bar{r}, \\
0=k+r f+s g=\bar{k}+\bar{s} f+\bar{r} g, \\
k^{2}+r^{2} f+s^{2} g=k v, \\
k \bar{k}+r \bar{s} f+s \bar{r} g=0, \\
\bar{k}^{2}+\bar{s}^{2} f+\bar{r}^{2} g=\bar{k} v, \\
f=\frac{(v-1) s+k}{s-r}, \quad g=\frac{(v-1) r+k}{r-s}, \\
f g=\frac{k \bar{k} v}{(r-s)^{2}} .
\end{gathered}
$$

The proofs of (7)-(13) are straightforward: (7) is clear; (8) is already mentioned and is immediate from $I+A+\bar{A}=J$; (9)-(12) are the values of $\operatorname{tr}(A), \operatorname{tr}(\bar{A}), \operatorname{tr}\left(A^{2}\right)$, $\operatorname{tr}(A \bar{A})$, and $\operatorname{tr}\left(\bar{A}^{2}\right)$; (13) follows from (7) and (9). To show (14), first restrict (4) to $\mathbb{U}_{\mathfrak{r}}$ and $\mathbb{U}_{\mathfrak{s}}$ and use (8) to find that $r$ and $s$ are the solutions of the quadratic equation

$$
\xi^{2}-(\lambda-\mu) \xi+\mu-k=0
$$

in indeterminate $\xi$, so that we have

$$
r+s=\lambda-\mu, \quad r s=\mu-k .
$$

Next, count the triples of distinct vertices $x, y, z$ such that $x \sim y \sim z \nsim x$ (in $G$ ) in two ways to get

$$
k(k-1-\lambda)=\bar{k} \mu .
$$

Then use (13) together with (7), (15), and (16).

Lemma 3.1. If $r$ and $s$ are non-integral then $f=g$ and we have

$$
v=4 \ell+1, \quad k=2 \ell, \quad \lambda=\ell-1, \quad \mu=\ell
$$

for some positive integer $\ell$. Moreover, in this case we have

$$
r=\frac{-1+\sqrt{1+4 \ell}}{2}, \quad s=\frac{-1-\sqrt{1+4 \ell}}{2} .
$$

Proof. See [3, p. 118]. We have $f=g$ since $r$ and $s$ are algebraic conjugates. Using (13) and (15), we then have $(v-1)(\mu-\lambda)=2 k$. Since $k<v-1$, this is possible only when $\mu-\lambda=1$ and $v-1=2 k$. In particular, we have $\bar{k}=k$ by (7), and hence it follows from (16) that $k=2 \mu$, as desired.

Strongly regular graphs with parameters of the form (17) are called conference graphs.

We say that $G$ is imprimitive if either $G$ or $\bar{G}$ is disconnected, and primitive otherwise. Thus, $G$ is imprimitive if and only if $G=p K_{q}$ or $G=K_{p \times q}$ for some integers $p, q \geqslant 2$. 
Example 3.2 (imprimitive graphs). Let $p$ and $q$ be integers such that $p, q \geqslant 2$. The disjoint union $p K_{q}$ is strongly regular with parameters $(p q, q-1, q-2,0)$ and restricted eigenvalues $r=q-1, s=-1$. The complete multipartite graph $K_{p \times q}$ is strongly regular with parameters $(p q,(p-1) q,(p-2) q,(p-1) q)$ and restricted eigenvalues $r=0, s=-q$.

We now introduce two more families of strongly regular graphs. See 3 , Sections 9.1.10-9.1.13]. Recall that an incidence structure is a triple $(\mathscr{P}, \mathscr{B}, \mathscr{I})$, where $\mathscr{P}$ and $\mathscr{B}$ are finite sets whose elements are called points and blocks, respectively, and where $\mathscr{I} \subset \mathscr{P} \times \mathscr{B}$. If $(p, b) \in \mathscr{I}$ then we say that $p$ and $b$ are incident, or $p$ is contained in $b$, and so on. The block graph of $(\mathscr{P}, \mathscr{B}, \mathscr{I})$ is the graph $G=(V, E)$ with $V=\mathscr{B}$ where two distinct blocks are adjacent if and only if they contain a point in common.

Example 3.3 (Steiner graphs). Let $m$ and $d$ be integers such that $2 \leqslant m<d$. A Steiner system $S(2, m, d)$ is an incidence structure $(\mathscr{P}, \mathscr{B}, \mathscr{I})$ with $|\mathscr{P}|=d$ such that every block contains precisely $m$ points, and that any two distinct points are contained in a unique block. The block graph of an $S(2, m, d)$ is called a Steiner graph and is strongly regular with parameters $(v, k, \lambda, \mu)$ provided that $v>d$, where

$$
v=\frac{d(d-1)}{m(m-1)}, \quad k=\frac{(d-m) m}{m-1}, \quad \lambda=(m-1)^{2}+\frac{d-2 m+1}{m-1}, \quad \mu=m^{2},
$$

and with restricted eigenvalues

$$
r=\frac{d-m^{2}}{m-1}, \quad s=-m
$$

Example 3.4 (Latin square graphs). Let $m$ and $e$ be integers with $m, e \geqslant 2$. A transversal design $T D(m, e)$ is an incidence structure $(\mathscr{P}, \mathscr{B}, \mathscr{I})$ where the point set is given a partition $\mathscr{P}=\mathscr{P}_{1} \sqcup \cdots \sqcup \mathscr{P}_{m}$ into $m$ groups of the same size $e$ (so $|\mathscr{P}|=m e)$, such that every block is incident with every group in exactly one point, and that any two points from distinct groups are contained in a unique block. The block graph of a $T D(m, e)$ is called a Latin square graph and is strongly regular with parameters $(v, k, \lambda, \mu)$ provided that $m \leqslant e$, where

$$
v=e^{2}, \quad k=m(e-1), \quad \lambda=(m-1)(m-2)+e-2, \quad \mu=m(m-1),
$$

and with restricted eigenvalues

$$
r=e-m, \quad s=-m .
$$

The following fundamental result is due to Neumaier [20].

Proposition 3.5 (20]). For any fixed integer $m>0$, there are only finitely many primitive strongly regular graphs with least eigenvalue $s=-m$, other than Steiner graphs and Latin square graphs.

\section{Proof of Theorem 2.1}

To prove Theorem 2.1, we invoke Lévy's continuity theorem concerning the pointwise convergence of the characteristic functions; see e.g., [1, Theorem 8.8.1]. Thus, we fix $\left(\xi_{1}, \xi_{2}\right) \in \mathbb{R}^{2}$ throughout the proof.

Recall that $G$ depends on $n$ in general. Let $\boldsymbol{A}$ and $\overline{\boldsymbol{A}}$ be the adjacency matrices of $G^{\square n}$ and $\bar{G}^{\square n}$, respectively. For convenience, let

$$
\Lambda_{n}=\{(j, h): j, h=0,1, \ldots, n, j+h \leqslant n\} .
$$


For every $(j, h) \in \Lambda_{n}$, consider the subspace

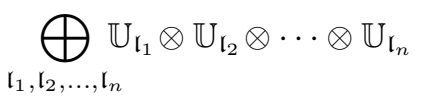

of $\left(\mathbb{C}^{v}\right)^{\otimes n} \cong \mathbb{C}^{v^{n}}$, where the sum is over $\mathfrak{l}_{1}, \mathfrak{l}_{2}, \ldots, \mathfrak{l}_{n} \in\{\mathfrak{k}, \mathfrak{r}, \mathfrak{s}\}$ such that

$$
\left\{\mathfrak{l}_{1}, \mathfrak{l}_{2}, \ldots, \mathfrak{l}_{n}\right\}=\{\underbrace{\mathfrak{k}, \ldots, \mathfrak{k}}_{n-j-h}, \underbrace{\mathfrak{r}, \ldots, \mathfrak{r}}_{j}, \underbrace{\mathfrak{s}, \ldots, \mathfrak{s}}_{h}\}
$$

as multisets. It has dimension

$$
\left(\begin{array}{c}
n \\
n-j-h, j, h
\end{array}\right) f^{j} g^{h}
$$

By virtue of (3) (and the corresponding formula for $\overline{\boldsymbol{A}}$ ), this subspace is a common eigenspact 5 of $(\boldsymbol{A}, \overline{\boldsymbol{A}})$ with eigenvalues $\left(\theta_{j, h}, \bar{\theta}_{j, h}\right)$, where

$$
\theta_{j, h}=(n-j-h) k+j r+h s, \quad \bar{\theta}_{j, h}=(n-j-h) \bar{k}+j \bar{s}+h \bar{r} .
$$

Note that $G^{\square n}$ and $\bar{G}^{\square n}$ are $n k$-regular and $n \bar{k}$-regular, respectively. Hence it follows from the above comments that the value of the characteristic function of $\nu_{G} \square n, \bar{G} \square n$ at $\left(\xi_{1}, \xi_{2}\right)$ is given by

$$
\begin{aligned}
\varphi_{\operatorname{tr}}\left(\exp \left(\frac{i \xi_{1} \boldsymbol{A}}{\sqrt{n k}}+\frac{i \xi_{2} \overline{\boldsymbol{A}}}{\sqrt{n \bar{k}}}\right)\right) \\
=\frac{1}{v^{n}} \sum_{(j, h) \in \Lambda_{n}} \exp \left(\frac{i \xi_{1} \theta_{j, h}}{\sqrt{n k}}+\frac{i \xi_{2} \bar{\theta}_{j, h}}{\sqrt{n \bar{k}}}\right)\left(\begin{array}{c}
n \\
n-j-h, j, h
\end{array}\right) f^{j} g^{h} \\
=\frac{1}{v^{n}} \sum_{(j, h) \in \Lambda_{n}}\left(e^{\Delta_{\mathfrak{k}}}\right)^{n-j-h}\left(f e^{\Delta_{\mathfrak{r}}}\right)^{j}\left(g e^{\Delta_{\mathfrak{s}}}\right)^{h}\left(\begin{array}{c}
n \\
n-j-h, j, h
\end{array}\right) \\
=\left(\frac{1}{v} e^{\Delta_{\mathfrak{k}}}+\frac{f}{v} e^{\Delta_{\mathfrak{r}}}+\frac{g}{v} e^{\Delta_{\mathfrak{s}}}\right)^{n} \\
=\exp \left(n \log \left(\frac{1}{v} e^{\Delta_{\mathfrak{k}}}+\frac{f}{v} e^{\Delta_{\mathfrak{r}}}+\frac{g}{v} e^{\Delta_{\mathfrak{s}}}\right)\right),
\end{aligned}
$$

where

$$
\Delta_{\mathfrak{k}}=\frac{i \xi_{1} k}{\sqrt{n k}}+\frac{i \xi_{2} \bar{k}}{\sqrt{n \bar{k}}}, \quad \Delta_{\mathfrak{r}}=\frac{i \xi_{1} r}{\sqrt{n k}}+\frac{i \xi_{2} \bar{s}}{\sqrt{n \bar{k}}}, \quad \Delta_{\mathfrak{s}}=\frac{i \xi_{1} s}{\sqrt{n k}}+\frac{i \xi_{2} \bar{r}}{\sqrt{n \bar{k}}} .
$$

Note by (8) that

$$
\frac{\bar{r}}{n} \rightarrow-\sigma, \quad \frac{\bar{s}}{n} \rightarrow-\rho
$$

and by (6) that

$$
-\min \{\kappa, \bar{\kappa}\} \leqslant \sigma \leqslant 0 \leqslant \rho \leqslant \min \{\kappa, \bar{\kappa}\}
$$

\footnotetext{
${ }^{5}$ It will turn out that the pairs $\left(\theta_{j, h}, \bar{\theta}_{j, h}\right)\left((j, h) \in \Lambda_{n}\right)$ are mutually distinct, and that these subspaces are indeed the maximal common eigenspaces of $(\boldsymbol{A}, \overline{\boldsymbol{A}})$; see Section 6 However, this fact is not necessary in the computation of (22) below.
} 
4.1. The case $\rho>0$ or $\sigma<0$. First we consider the case where $\rho>0$ or $\sigma<0$. Then we have $\kappa, \bar{\kappa}>0$ by (25), and moreover $1 / v=O(1 / n)$. Note that each of $\Delta_{\mathfrak{k}}$, $\Delta_{\mathfrak{r}}$, and $\Delta_{\mathfrak{s}}$ converges by (24). On the one hand, by (14) we have

$$
\frac{f g}{n} \rightarrow \frac{\kappa \bar{\kappa} \omega}{(\rho-\sigma)^{2}}<\infty
$$

so that

On the other hand, by (13) we have

$$
\frac{f g}{n^{2}} \rightarrow 0 .
$$

$$
\frac{f}{n} \rightarrow \frac{\omega \sigma}{\sigma-\rho}, \quad \frac{g}{n} \rightarrow \frac{\omega \rho}{\rho-\sigma} .
$$

Hence we have $\rho=0$ or $\sigma=0$.

For the moment, assume that $\rho=0$ and $\sigma<0$, so that

$$
\frac{f}{n} \rightarrow \omega, \quad \frac{g}{n} \rightarrow 0 .
$$

Then it follows from (26) that

$$
g \rightarrow g_{\infty}:=\frac{\kappa \bar{\kappa}}{\sigma^{2}}
$$

In particular, $g$ is bounded. Moreover, by (9) we have

$$
r=-\frac{k+s g}{f} \rightarrow r_{\infty}:=-\frac{\kappa+\sigma g_{\infty}}{\omega},
$$

so that $r$ and $\bar{s}=-r-1$ are also bounded, and thus $\Delta_{\mathfrak{r}}=O(1 / n)$. Since by (7)

$$
\frac{f}{v} e^{\Delta_{\mathfrak{r}}}=e^{\Delta_{\mathfrak{r}}}-\frac{1+g}{v} e^{\Delta_{\mathfrak{r}}}=1+\Delta_{\mathfrak{r}}-\frac{1+g}{v}+O\left(\frac{1}{n^{2}}\right),
$$

it follows using (24) that (22) equals

$$
\begin{aligned}
\exp \left(n\left(\frac{1}{v} e^{\Delta_{\mathfrak{k}}}+\frac{g}{v} e^{\Delta_{\mathfrak{s}}}+\Delta_{\mathfrak{r}}-\frac{1+g}{v}+O\left(\frac{1}{n^{2}}\right)\right)\right) & \\
= & \exp \left(\frac{n}{v} e^{\Delta_{\mathfrak{t}}}+\frac{n g}{v} e^{\Delta_{\mathfrak{s}}}+n \Delta_{\mathfrak{r}}-\frac{n(1+g)}{v}+O\left(\frac{1}{n}\right)\right) \\
\rightarrow & \exp \left(\exp \left(i \xi_{1} \sqrt{\kappa}+i \xi_{2} \sqrt{\bar{\kappa}}\right) \frac{1}{\omega}+\exp \left(\frac{i \xi_{1} \sigma}{\sqrt{\kappa}}-\frac{i \xi_{2} \sigma}{\sqrt{\bar{\kappa}}}\right) \frac{g_{\infty}}{\omega}\right. \\
& \left.+\frac{i \xi_{1} r_{\infty}}{\sqrt{\kappa}}-\frac{i \xi_{2}\left(r_{\infty}+1\right)}{\sqrt{\bar{\kappa}}}-\frac{1+g_{\infty}}{\omega}\right) .
\end{aligned}
$$

We note that the limit in (27) is the value at $\left(\xi_{1}, \xi_{2}\right)$ of the characteristic function of an affine transformation of a bivariate Poisson distribution.

We now show that $G$ is a complete multipartite graph for $n \gg 0$, so that we have $\sigma=-\bar{\kappa}, r_{\infty}=0, g_{\infty}=\kappa / \bar{\kappa}$, and the limit in (27) becomes

$$
\exp \left(\exp \left(i \xi_{1} \sqrt{\kappa}+i \xi_{2} \sqrt{\bar{\kappa}}\right) \frac{1}{\omega}+\exp \left(-\frac{i \xi_{1} \bar{\kappa}}{\sqrt{\kappa}}+i \xi_{2} \sqrt{\bar{\kappa}}\right) \frac{\kappa}{\omega \bar{\kappa}}-\frac{i \xi_{2}}{\sqrt{\bar{\kappa}}}-\frac{1}{\bar{\kappa}}\right)
$$

which corresponds to the distribution $\nu$ given in Theorem 2.1](i). Recall that $\bar{s}$ is bounded. By virtue of Proposition 3.5 and Lemma 3.1, $\bar{G}$ is one of the following for $n \gg 0$ : $\left(\bar{G}_{1}\right)$ a conference graph; $\left(\bar{G}_{2}\right)$ a disjoint union $p K_{q}$ of complete graphs; $\left(\bar{G}_{3}\right)$ a complete multipartite graph $K_{p \times q} ;\left(\bar{G}_{4}\right)$ a Steiner graph of an $S(2, m, d)$; 
$\left(\bar{G}_{5}\right)$ a Latin square graph of a $T D(m, e)$. Case $\left(\bar{G}_{1}\right)$ is impossible as $v$ would also be bounded. For Case $\left(\bar{G}_{3}\right)$, we have $\sigma=0$, a contradiction. If $\bar{G}$ is a Steiner graph of an $S(2, m, d)$ as in Case $\left(\bar{G}_{4}\right)$, then $m$ is bounded since $\bar{s}=-m$. However, since $\bar{k}$ and $v$ are linear and quadratic in $d$, respectively, $\bar{\kappa}$ and $\omega$ cannot be both finite and non-zero, a contradiction. The same argument shows that Case $\left(\bar{G}_{5}\right)$ is also impossible. Hence we are left with Case $\left(\bar{G}_{2}\right)$, so that we have (i) in Theorem 2.1 .

If $\rho>0$ and $\sigma=0$, then switching the roles of $G$ and $\bar{G}$ gives (ii) in Theorem 2.1. This completes the case where $\rho>0$ or $\sigma<0$.

4.2. The case $\rho=\sigma=0$. Next we deal with the case where $\rho=\sigma=0$. Note that $\Delta_{\mathfrak{k}}$ converges, and that $\Delta_{\mathfrak{r}}, \Delta_{\mathfrak{s}} \rightarrow 0$ in view of (6) and (24). From (10), (11), and (12) it follows that

$$
r^{2} f \leqslant k v, \quad-r \bar{s} f \leqslant k \bar{k}, \quad \bar{s}^{2} f \leqslant \bar{k} v,
$$

from which it follows that

$$
\left|\Delta_{\mathfrak{r}}^{2}\right| f \leqslant\left(\frac{\xi_{1}^{2} r^{2}}{n k}-2 \frac{\left|\xi_{1} \xi_{2}\right| r \bar{s}}{n \sqrt{k \bar{k}}}+\frac{\xi_{2}^{2} \bar{s}^{2}}{n \bar{k}}\right) f \leqslant \frac{\xi_{1}^{2} v}{n}+2 \frac{\left|\xi_{1} \xi_{2}\right| \sqrt{k \bar{k}}}{n}+\frac{\xi_{2}^{2} v}{n} .
$$

Hence $\Delta_{\mathfrak{r}}^{2} f$ is bounded. Likewise, we can show that $\Delta_{\mathfrak{s}}^{2} g$ is bounded. We also need the following identities which are immediate from (9)-(12):

$$
\begin{gathered}
\Delta_{\mathfrak{k}}+\Delta_{\mathfrak{r}} f+\Delta_{\mathfrak{s}} g=0, \\
\Delta_{\mathfrak{k}}^{2}+\Delta_{\mathfrak{r}}^{2} f+\Delta_{\mathfrak{s}}^{2} g=-\frac{\xi_{1}^{2} v}{n}-\frac{\xi_{2}^{2} v}{n} .
\end{gathered}
$$

For the moment, assume that $\kappa>0$ or $\bar{\kappa}>0$. Note that $1 / v=O(1 / n)$ in this case. Moreover, we have

$$
\frac{f}{v} e^{\Delta_{\mathfrak{r}}}=\left(1+\Delta_{\mathfrak{r}}+\frac{\Delta_{\mathfrak{r}}^{2}}{2}+O\left(\Delta_{\mathfrak{r}}^{3}\right)\right) \frac{f}{v}=\left(1+\Delta_{\mathfrak{r}}+\frac{\Delta_{\mathfrak{r}}^{2}}{2}\right) \frac{f}{v}+o\left(\frac{1}{n}\right),
$$

and similarly for $g e^{\Delta_{s}} / v$. Hence it follows from (7), (29), and (30) that (22) equals

$$
\begin{aligned}
\exp \left(n \operatorname { l o g } \left(\frac{1}{v} e^{\Delta_{\mathfrak{k}}}+\left(1+\Delta_{\mathfrak{r}}+\frac{\Delta_{\mathfrak{r}}^{2}}{2}\right) \frac{f}{v}\right.\right. & \left.\left.+\left(1+\Delta_{\mathfrak{s}}+\frac{\Delta_{\mathfrak{s}}^{2}}{2}\right) \frac{g}{v}+o\left(\frac{1}{n}\right)\right)\right) \\
=\exp \left(n \operatorname { l o g } \left(1+\frac{1}{v} e^{\Delta_{\mathfrak{k}}}-\frac{\Delta_{\mathfrak{k}}}{v}\right.\right. & \left.\left.+\left(\Delta_{\mathfrak{r}}^{2} f+\Delta_{\mathfrak{s}}^{2} g\right) \frac{1}{2 v}-\frac{1}{v}+o\left(\frac{1}{n}\right)\right)\right) \\
= & \exp \left(n\left(\frac{1}{v} e^{\Delta_{\mathfrak{k}}}-\frac{\Delta_{\mathfrak{k}}}{v}+\left(\Delta_{\mathfrak{r}}^{2} f+\Delta_{\mathfrak{s}}^{2} g\right) \frac{1}{2 v}-\frac{1}{v}+o\left(\frac{1}{n}\right)\right)\right) \\
\rightarrow & \exp \left(\exp \left(i \xi_{1} \sqrt{\kappa}+i \xi_{2} \sqrt{\bar{\kappa}}\right) \frac{1}{\omega}-\frac{i \xi_{1} \sqrt{\kappa}+i \xi_{2} \sqrt{\bar{\kappa}}}{\omega}\right. \\
& \left.-\frac{\left(\xi_{1} \sqrt{\bar{\kappa}}-\xi_{2} \sqrt{\kappa}\right)^{2}}{2 \omega}-\frac{1}{\omega}\right) .
\end{aligned}
$$

It is a straightforward matter to show that this corresponds to the distribution $\nu$ in Theorem 2.1(iii). 
Finally, assume that $\kappa=\bar{\kappa}=0$. In this case, we have $\omega=0$, i.e.,

$$
\frac{v}{n} \rightarrow 0
$$

Note that $\Delta_{\mathfrak{k}}, \Delta_{\mathfrak{r}}, \Delta_{\mathfrak{s}}=O\left((v / n)^{1 / 2}\right)$. Moreover, it follows from (28) that $\Delta_{\mathfrak{r}}^{2} f=$ $O(v / n)$, and likewise we have $\Delta_{\mathfrak{s}}^{2} g=O(v / n)$. Hence it follows from (77), (29), and (30) that (22) equals

$$
\begin{aligned}
\exp ( & n \log \left(\left(1+\Delta_{\mathfrak{k}}+\frac{\Delta_{\mathfrak{k}}^{2}}{2}\right) \frac{1}{v}+\left(1+\Delta_{\mathfrak{r}}+\frac{\Delta_{\mathfrak{r}}^{2}}{2}\right) \frac{f}{v}\right. \\
& \left.\left.+\left(1+\Delta_{\mathfrak{s}}+\frac{\Delta_{\mathfrak{s}}^{2}}{2}\right) \frac{g}{v}+O\left(\left(\frac{v}{n}\right)^{\frac{3}{2}}\right) \frac{1}{v}\right)\right) \\
= & \exp \left(n \log \left(1-\frac{\xi_{1}^{2}}{2 n}-\frac{\xi_{2}^{2}}{2 n}+O\left(\left(\frac{v}{n}\right)^{\frac{3}{2}}\right) \frac{1}{v}\right)\right) \\
= & \exp \left(n\left(-\frac{\xi_{1}^{2}}{2 n}-\frac{\xi_{2}^{2}}{2 n}+O\left(\left(\frac{v}{n}\right)^{\frac{3}{2}}\right) \frac{1}{v}\right)\right) \\
= & \exp \left(-\frac{\xi_{1}^{2}}{2}-\frac{\xi_{2}^{2}}{2}+O\left(\left(\frac{v}{n}\right)^{\frac{1}{2}}\right)\right) \\
\rightarrow & \exp \left(-\frac{\xi_{1}^{2}}{2}-\frac{\xi_{2}^{2}}{2}\right) .
\end{aligned}
$$

This corresponds to the standard bivariate Gaussian distribution, and hence we have (iv) in Theorem 2.1 .

This completes the proof of Theorem 2.1.

\section{EXAmPles}

The graph $G$ (which we recall is a function in $n$ ) is already identified for (i) and (ii) in Theorem 2.1, whereas (iv) is a degenerate case and is easily realized as it only requires $v / n \rightarrow 0$. Below are some examples for (iii) in Theorem 2.1, i.e., such that $\kappa>0$ or $\bar{\kappa}>0$, and $\rho=\sigma=0$.

Example 5.1. Consider the imprimitive strongly regular graphs $p K_{q}$ and $K_{p \times q}$. Assume that $p q$ is (essentially) linear in $n$ and that $q / n \rightarrow 0$. Then we have $\kappa=0$ and $\bar{\kappa}>0$ in Theorem 2.1(iii) for $p K_{q}$, and $\kappa>0$ and $\bar{\kappa}=0$ for $K_{p \times q}$.

Example 5.2 (Paley graphs). Let $q$ be a prime power with $q \equiv 1(\bmod 4)$. The Paley graph Paley $(q)$ has vertex set $\mathbb{F}_{q}$ (the finite field with $q$ elements), where two distinct vertices are adjacent if and only if their difference is a square. It is easy to see that $\operatorname{Paley}(q)$ is a conference graph. See [3. Sections 9.1.1 and 9.1.2]. Hence if we take $n$ to be linear in $q$, then it follows from (17) and (18) that we are in Theorem 2.1(iii) with $\kappa=\bar{\kappa}>0$.

Example 5.3 (Symplectic graphs). Let $q$ be a prime power, and let $\ell \geqslant 2$ be an integer. We endow $\mathbb{F}_{q}^{2 \ell}$ with a non-degenerate symplectic form. The Symplectic graph $S p_{2 \ell}(q)$ has as vertex set the set of one-dimensional subspaces (i.e., projective points) of $\mathbb{F}_{q}^{2 \ell}$, where two distinct vertices are adjacent if and only if they are orthogonal. The graph $S p_{2 \ell}(q)$ is strongly regular with parameters $(v, k, \lambda, \mu)$, where

$$
v=\frac{q^{2 \ell}-1}{q-1}, \quad k=\frac{q^{2 \ell-1}-q}{q-1}, \quad \lambda=\frac{q^{2 \ell-2}-2 q+1}{q-1}, \quad \mu=\frac{q^{2 \ell-2}-1}{q-1},
$$


and with restricted eigenvalues

$$
r=q^{\ell-1}-1, \quad s=-q^{\ell-1}-1 .
$$

Fix $q$ and let $\ell \rightarrow \infty$. If $n$ is linear in $q^{2 \ell}$ then again we are in Theorem 2.1(iii) with $\kappa=\bar{\kappa} /(q-1)>0$. There are many other infinite families of strongly regular graphs related to finite geometry; see [2] and [3, Section 9.9].

Example 5.4. Let $q$ be a prime power. Let $H_{1}, H_{2}, \ldots, H_{m}$ be distinct onedimensional subspaces of $\mathbb{F}_{q}^{2}$, where $1 \leqslant m \leqslant q$. For $j=1,2, \ldots, m$, let $\mathscr{P}_{j}$ be the set of $q$ parallel affine subspaces of $\mathbb{F}_{q}^{2}$ with direction $H_{j}$, i.e.,

$$
\mathscr{P}_{j}=\left\{H_{j}+x: x \in \mathbb{F}_{q}^{2}\right\} \quad(j=1,2, \ldots, m) .
$$

Let $\mathscr{P}=\mathscr{P}_{1} \sqcup \cdots \sqcup \mathscr{P}_{m}$ and $\mathscr{B}=\mathbb{F}_{q}^{2}$. Consider the incidence structure $(\mathscr{P}, \mathscr{B}, \mathscr{I})$, where a point $H_{j}+x$ and a block $y$ are incident if and only if $y \in H_{j}+x$. Then it is easy to see that $(\mathscr{P}, \mathscr{B}, \mathscr{I})$ is a $T D(m, q)$. Hence if we take both $q^{2}$ and $m q$ to be linear in $n$, then the corresponding Latin square graph attains Theorem 2.1(iii) with $\kappa>0$. We may view $\operatorname{Paley}\left(q^{2}\right)$ (for odd $q$ ) in this way with $m=(q+1) / 2$, as $\mathbb{F}_{q^{2}} \cong \mathbb{F}_{q}^{2}$. We note that, unlike the previous examples, any $\kappa, \bar{\kappa} \geqslant 0$ can be achieved here as limits. There is also a more general construction of strongly regular graphs from cyclotomy, all giving rise to examples of Theorem 2.1(iii); cf. [3. Section 9.8.5].

\section{Bivariate Charlier-Hermite polynomials}

It is more natural to understand our approach of considering the pair $\left(G^{\square n}, \bar{G}^{\square n}\right)$ with $G$ and $\bar{G}$ both strongly regular, in terms of association schemes; cf. [3, Chapter 11]. An association scheme with $d$ classes is an edge decomposition of a complete graph $K_{v}$ into $d$ graphs $G_{1}, G_{2}, \ldots, G_{d}$, whose adjacency matrices $A_{1}, A_{2}, \ldots, A_{d}$, together with $A_{0}:=I$, form a basis of a $(d+1)$-dimensional matrix *-algebra $M$ over $\mathbb{C}$, called the Bose-Mesner algebra. Thus, the association schemes with one class are the same thing as the complete graphs, and those with two classes are the pairs of strongly regular graphs and their complements (cf. (4)). Since $M$ consists of symmetric matrices only, it follows that $M$ is commutative and has precisely $d+1$ maximal common eigenspaces.

With the notation in the previous sections, we now consider the association scheme $G, \bar{G}$ with Bose-Mesner algebra $M=\langle I, A, \bar{A}\rangle$. Observe that the $n^{\text {th }}$ symmetric tensor space $\operatorname{Sym}^{n}(M)$ of $M$ is again the Bose-Mesner algebra of another association scheme consisting of the graphs on $V^{n}$ with adjacency matrices (cf. (19))

$$
\boldsymbol{A}_{a, b}=\sum_{B_{1}, B_{2}, \ldots, B_{n}} B_{1} \otimes B_{2} \otimes \cdots \otimes B_{n} \quad\left((a, b) \in \Lambda_{n}\right),
$$

where the sum is over $B_{1}, B_{2}, \ldots, B_{n} \in\{I, A, \bar{A}\}$ such that

$$
\left\{B_{1}, B_{2}, \ldots, B_{n}\right\}=\{\underbrace{I, \ldots, I}_{n-a-b}, \underbrace{A, \ldots, A}_{a}, \underbrace{\bar{A}, \ldots, \bar{A}}_{b}\}
$$

as multisets; cf. [5, Section 2.5]. In particular, we have (cf. (3) )

$$
\boldsymbol{A}=\boldsymbol{A}_{1,0}, \quad \overline{\boldsymbol{A}}=\boldsymbol{A}_{0,1} .
$$

Moreover, the subspaces (20) for $(j, h) \in \Lambda_{n}$ are the maximal common eigenspaces of $\operatorname{Sym}^{n}(M)$. Mizukawa and Tanaka 18] showed that the corresponding eigenvalues 
of $\boldsymbol{A}_{a, b}$ are expressed as

$$
k_{a, b} \mathcal{K}_{a, b}(j, h) \quad\left((j, h) \in \Lambda_{n}\right)
$$

where

$$
k_{a, b}=\left(\begin{array}{c}
n \\
n-a-b, a, b
\end{array}\right) k^{a} \bar{k}^{b}
$$

denotes the degree of the graph with adjacency matrix $\boldsymbol{A}_{a, b}$, and $\mathcal{K}_{a, b}(j, h)$ is the terminating Aomoto-Gelfand hypergeometric series

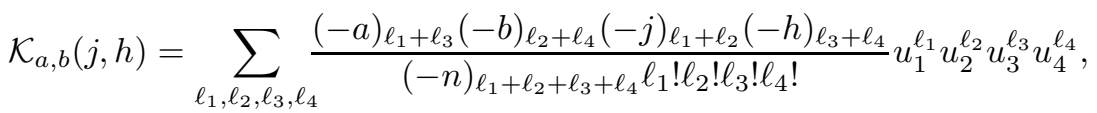

where the sum is over $\ell_{1}, \ell_{2}, \ell_{3}, \ell_{4}=0,1, \ldots, n$ with $\ell_{1}+\ell_{2}+\ell_{3}+\ell_{4} \leqslant n$, and

$$
u_{1}=1-\frac{r}{k}, \quad u_{2}=1-\frac{\bar{s}}{\bar{k}}, \quad u_{3}=1-\frac{s}{k}, \quad u_{4}=1-\frac{\bar{r}}{\bar{k}} .
$$

We note that $6 \mathcal{K}_{a, b}(j, h)$ is a polynomial in $j$ and $h$ with (total) degree $a+b$. The $\mathcal{K}_{a, b}$ are the bivariate Krawtchouk polynomials and are also known as the Rahman polynomials; cf. [9, 10, 13, 17. For $(j, h) \in \Lambda_{n}$, we have (cf. [8, 9, 21])

$$
\begin{aligned}
\sum_{(a, b) \in \Lambda_{n}} k_{a, b} \mathcal{K}_{a, b}(j, h) \xi_{1}^{a} \xi_{2}^{b} & \\
& =\left(1+k \xi_{1}+\bar{k} \xi_{2}\right)^{n-j-h}\left(1+r \xi_{1}+\bar{s} \xi_{2}\right)^{j}\left(1+s \xi_{1}+\bar{r} \xi_{2}\right)^{h},
\end{aligned}
$$

where $\xi_{1}$ and $\xi_{2}$ are indeterminates. It should be remarked that, if $j$ and $h$ are also indeterminates, then the RHS above belongs to the formal power series ring $\mathbb{C}[j, h]\left[\left[\xi_{1}, \xi_{2}\right]\right]$ over the polynomial ring $\mathbb{C}[j, h]$, and the coefficients of $\xi_{1}^{a} \xi_{2}^{b}$ on both sides still agree whenever $a+b \leqslant n$.

The goal of this section is to construct a family of bivariate hypergeometric orthogonal polynomials with respect to the probability measure $\nu$ in Theorem 2.1(iii) as limits of the $\mathcal{K}_{a, b}$, so that we will assume from now on that

$$
\omega=\kappa+\bar{\kappa}>0, \quad \rho=\sigma=0 .
$$

(For the other cases in Theorem 2.1 similar discussions give rise to the bivariate Charlier and Hermite polynomials; see Remark 6.1 below.) For the convergence, however, we will work with the normalization $\sqrt{k_{a, b}} \mathcal{K}_{a, b}$ which gives the eigenvalues of $\boldsymbol{A}_{a, b} / \sqrt{k_{a, b}}$. Moreover, we also make the change of variables (cf. (21))

$$
x=\frac{\theta_{j, h}}{\sqrt{n k}}, \quad y=\frac{\bar{\theta}_{j, h}}{\sqrt{n \bar{k}}}
$$

or equivalently,

$$
\begin{aligned}
& j=\frac{(\bar{k}-\bar{r}) \sqrt{n k}}{v(r-s)} x-\frac{(k-s) \sqrt{n \bar{k}}}{v(r-s)} y+\frac{n f}{v}, \\
& h=\frac{(\bar{k}-\bar{s}) \sqrt{n k}}{v(s-r)} x-\frac{(k-r) \sqrt{n \bar{k}}}{v(s-r)} y+\frac{n g}{v},
\end{aligned}
$$

\footnotetext{
${ }^{6}$ This in particular shows that $\operatorname{Sym}^{n}(M)$ is generated by $\boldsymbol{A}$ and $\overline{\boldsymbol{A}}$ which correspond to linear polynomials, so that the subspaces (20) are also the maximal common eigenspaces of $(\boldsymbol{A}, \overline{\boldsymbol{A}})$.
} 
where we have used (77), (8), and (13). Thus, we define the bivariate polynomials $\hat{\mathcal{K}}_{a, b}(x, y)$ by

$$
\hat{\mathcal{K}}_{a, b}(x, y)=\sqrt{k_{a, b}} \mathcal{K}_{a, b}(j, h),
$$

where the variables $(j, h)$ and $(x, y)$ are related as above. Then, the $\hat{\mathcal{K}}_{a, b}$ satisfy the following orthogonality relation:

$$
\int_{\mathbb{R}^{2}} \hat{\mathcal{K}}_{a, b}(x, y) \hat{\mathcal{K}}_{c, d}(x, y) \nu_{G^{\square n}, \bar{G} \square n}(d x d y)=\varphi_{\operatorname{tr}}\left(\frac{\boldsymbol{A}_{a, b} \boldsymbol{A}_{c, d}}{\sqrt{k_{a, b} k_{c, d}}}\right)=\delta_{a, c} \delta_{b, d}
$$

for $(a, b),(c, d) \in \Lambda_{n}$. We also note that

$$
\hat{\mathcal{K}}_{1,0}(x, y)=x, \quad \hat{\mathcal{K}}_{0,1}(x, y)=y .
$$

Recall $\Delta_{\mathfrak{k}}, \Delta_{\mathfrak{r}}$, and $\Delta_{\mathfrak{s}}$ from (23), where $\xi_{1}$ and $\xi_{2}$ are indeterminates in the present context, rather than real scalars. To compute the limits of the $\hat{\mathcal{K}}_{a, b}$, we consider instead of (34)

$$
\left(1-i \Delta_{\mathfrak{k}}\right)^{n-j-h}\left(1-i \Delta_{\mathfrak{r}}\right)^{j}\left(1-i \Delta_{\mathfrak{s}}\right)^{h},
$$

which is an element of $\mathbb{C}[x, y]\left[\left[\xi_{1}, \xi_{2}\right]\right]$ via (36) and (37), where we view $x$ and $y$ as indeterminates. On the one hand, in view of the remark after (34), the terms in (40) of degree at most $n$ (in $\xi_{1}$ and $\xi_{2}$ ) are

$$
\frac{k_{a, b} \mathcal{K}_{a, b}(j, h)}{\sqrt{n^{a+b} k^{a} \bar{k}^{b}}} \xi_{1}^{a} \xi_{2}^{b}=\sqrt{\frac{k_{a, b}}{n^{a+b} k^{a} \bar{k}^{b}}} \hat{\mathcal{K}}_{a, b}(x, y) \xi_{1}^{a} \xi_{2}^{b} \quad\left((a, b) \in \Lambda_{n}\right),
$$

where by (33) we have

$$
\frac{k_{a, b}}{n^{a+b} k^{a} \bar{k}^{b}} \rightarrow \frac{1}{a ! b !}
$$

for fixed $a, b=0,1,2, \ldots$ On the other hand, since

$$
n-j-h=\frac{\sqrt{n k} x+\sqrt{n \bar{k}} y+n}{v}
$$

by (77) and (8), we have

$$
\left(1-i \Delta_{\mathfrak{k}}\right)^{n-j-h} \rightarrow\left(1+\sqrt{\kappa} \xi_{1}+\sqrt{\bar{\kappa}} \xi_{2}\right)^{(\sqrt{\kappa} x+\sqrt{\bar{\kappa}} y+1) / \omega}
$$

as elements of $\mathbb{C}[x, y]\left[\left[\xi_{1}, \xi_{2}\right]\right]$. The limit of the latter two factors of (401) can be computed in the same manner as in the proof of Theorem 2.1(iii); cf. Section 4.2 . Recall that $\Delta_{\mathfrak{r}}, \Delta_{\mathfrak{s}} \rightarrow 0$. Since the coefficients of $f \Delta_{\mathfrak{r}}^{2}$ and $g \Delta_{\mathfrak{s}}^{2}$ are bounded as in (28), we have

$$
f \Delta_{\mathfrak{r}}^{\ell}, g \Delta_{\mathfrak{s}}^{\ell} \rightarrow 0 \quad(\ell=3,4, \ldots) .
$$

Likewise, using (6) and (8), we can routinely show that

$$
\left(j-\frac{n f}{v}\right) \Delta_{\mathfrak{r}}^{\ell},\left(h-\frac{n g}{v}\right) \Delta_{\mathfrak{s}}^{\ell} \rightarrow 0 \quad(\ell=2,3, \ldots) .
$$


Since $\Delta_{\mathfrak{r}}^{\ell}, \Delta_{\mathfrak{s}}^{\ell}(\ell=1,2, \ldots)$ are homogeneous of degree $\ell$ in $\xi_{1}$ and $\xi_{2}$, it follows from (8), (29), and (30) that (cf. (31))

$$
\begin{aligned}
\left(1-i \Delta_{\mathfrak{r}}\right)^{j} & \left(1-i \Delta_{\mathfrak{s}}\right)^{h} \\
= & \exp \left(-j \sum_{\ell=1}^{\infty} \frac{\left(i \Delta_{\mathfrak{r}}\right)^{\ell}}{\ell}-h \sum_{\ell=1}^{\infty} \frac{\left(i \Delta_{\mathfrak{s}}\right)^{\ell}}{\ell}\right) \\
= & \exp \left(-\frac{n f}{v}\left(\left(i \Delta_{\mathfrak{r}}\right)+\frac{\left(i \Delta_{\mathfrak{r}}\right)^{2}}{2}\right)-\frac{n g}{v}\left(\left(i \Delta_{\mathfrak{s}}\right)+\frac{\left(i \Delta_{\mathfrak{s}}\right)^{2}}{2}\right)\right. \\
& \left.\quad\left(j-\frac{n f}{v}\right)\left(i \Delta_{\mathfrak{r}}\right)-\left(h-\frac{n g}{v}\right)\left(i \Delta_{\mathfrak{s}}\right)+o(1)\right) \\
= & \exp \left(\frac{n\left(i \Delta_{\mathfrak{k}}\right)}{v}-\frac{n}{2 v}\left(f\left(i \Delta_{\mathfrak{r}}\right)^{2}+g\left(i \Delta_{\mathfrak{s}}\right)^{2}\right)\right. \\
& \left.+\frac{(\sqrt{\bar{k}} x-\sqrt{k} y)\left(\sqrt{\bar{k}} \xi_{1}-\sqrt{k} \xi_{2}\right)}{v}+o(1)\right) \\
\rightarrow & \exp \left(-\frac{\sqrt{\kappa} \xi_{1}+\sqrt{\bar{\kappa}} \xi_{2}}{\omega}-\frac{\left(\sqrt{\bar{\kappa}} \xi_{1}-\sqrt{\kappa} \xi_{2}\right)^{2}}{2 \omega}\right. \\
+ & \left.\frac{(\sqrt{\kappa} x-\sqrt{\kappa} y)\left(\sqrt{\kappa} \xi_{1}-\sqrt{\kappa} \xi_{2}\right)}{\omega}\right) .
\end{aligned}
$$

From (40)-(44) it follows that there are polynomials $\mathcal{C H}_{a, b}(x, y)(a, b=0,1,2, \ldots)$ in $x$ and $y$ such that

$$
\hat{\mathcal{K}}_{a, b}(x, y) \rightarrow \mathcal{C H}_{a, b}(x, y) \quad(a, b=0,1,2, \ldots),
$$

and that the generating function

$$
\sum_{a, b=0}^{\infty} \frac{\mathcal{C H}_{a, b}(x, y)}{\sqrt{a ! b !}} \xi_{1}^{a} \xi_{2}^{b}
$$

equals the product of the limits in (43) and (44) as elements in $\mathbb{C}[x, y]\left[\left[\xi_{1}, \xi_{2}\right]\right]$. We call the $\mathcal{C H}_{a, b}$ the bivariate Charlier-Hermite polynomials. We note by (39) that

$$
\mathrm{CH}_{1,0}(x, y)=x, \quad \mathrm{CH}_{0,1}(x, y)=y .
$$

The $\mathcal{C} \mathcal{H}_{a, b}$ depend on two parameters $\kappa, \bar{\kappa} \geqslant 0$, where $\omega=\kappa+\bar{\kappa}>0$.

From the generating function we may derive an explicit formula for the $\mathcal{C H}_{a, b}$. Observe that the limit in (43) equals

$$
\sum_{\ell_{1}, \ell_{2}=0}^{\infty} \frac{\left(-\frac{\sqrt{\kappa} x+\sqrt{\bar{\kappa}} y+1}{\omega}\right)_{\ell_{1}+\ell_{2}}}{\ell_{1} ! \ell_{2} !}(-1)^{\ell_{1}+\ell_{2}}\left(\sqrt{\kappa} \xi_{1}\right)^{\ell_{1}}\left(\sqrt{\kappa} \xi_{2}\right)^{\ell_{2}},
$$

whereas the limit in (44) equals the product of the following two elements:

$$
\begin{gathered}
\sum_{\ell_{3}, \ell_{4}, \ell_{5}=0}^{\infty} \frac{1}{\ell_{3} ! \ell_{4} ! \ell_{5} !}\left(-\frac{\bar{\kappa} \xi_{1}^{2}}{2 \omega}\right)^{\ell_{3}}\left(-\frac{\kappa \xi_{2}^{2}}{2 \omega}\right)^{\ell_{4}}\left(\frac{\sqrt{\kappa \bar{\kappa}} \xi_{1} \xi_{2}}{\omega}\right)^{\ell_{5}}, \\
\sum_{\ell_{6}, \ell_{7}=0}^{\infty} \frac{1}{\ell_{6} ! \ell_{7} !}\left(\frac{\bar{\kappa} x-\sqrt{\kappa \bar{\kappa}} y-\sqrt{\kappa}}{\omega} \xi_{1}\right)^{\ell_{6}}\left(\frac{\kappa y-\sqrt{\kappa \bar{\kappa}} x-\sqrt{\bar{\kappa}}}{\omega} \xi_{2}\right)^{\ell_{7}} .
\end{gathered}
$$


Picking out the coefficient of $\xi_{1}^{a} \xi_{2}^{b}$ in the product of the above three elements and simplifying the result using

$$
\ell_{6}=a-\ell_{1}-2 \ell_{3}-\ell_{5}, \quad \ell_{7}=b-\ell_{2}-2 \ell_{4}-\ell_{5},
$$

it follows that $\mathcal{C H}_{a, b}(x, y)$ equals

$$
\frac{(\bar{\kappa} x-\sqrt{\kappa \bar{\kappa}} y-\sqrt{\kappa})^{a}(\kappa y-\sqrt{\kappa \bar{\kappa}} x-\sqrt{\bar{\kappa}})^{b}}{\sqrt{a ! b !} \omega^{a+b}}
$$

times

$$
\begin{gathered}
\sum_{\ell_{1}, \ell_{2}, \ell_{3}, \ell_{4}, \ell_{5}=0}^{\infty} \frac{(-a)_{\ell_{1}+2 \ell_{3}+\ell_{5}}(-b)_{\ell_{2}+2 \ell_{4}+\ell_{5}}\left(-\frac{\sqrt{\kappa} x+\sqrt{\kappa} y+1}{\omega}\right)_{\ell_{1}+\ell_{2}}}{\ell_{1} ! \ell_{2} ! \ell_{3} ! \ell_{4} ! \ell_{5} !} \\
\times \frac{\left(-\frac{1}{2}\right)^{\ell_{3}+\ell_{4}} \sqrt{\kappa} \ell_{1}^{\ell_{1}+2 \ell_{4}+\ell_{5}} \sqrt{\bar{\kappa}}^{\ell_{2}+2 \ell_{3}+\ell_{5}} \omega^{\ell_{1}+\ell_{2}+\ell_{3}+\ell_{4}+\ell_{5}}}{(\bar{\kappa} x-\sqrt{\kappa \bar{\kappa}} y-\sqrt{\kappa})^{\ell_{1}+2 \ell_{3}+\ell_{5}}(\kappa y-\sqrt{\kappa \bar{\kappa}} x-\sqrt{\bar{\kappa}})^{\ell_{2}+2 \ell_{4}+\ell_{5}}} .
\end{gathered}
$$

This expression is not of Aomoto-Gelfand type, but is still a terminating hypergeometric series in the sense of Horn.

We next obtain five-term recurrence relations for the $\mathcal{C} \mathcal{H}_{a, b}$ as limits of those for the $\hat{\mathcal{K}}_{a, b}$. Note that

$$
A \bar{A}=A(J-A-I)=(\bar{k}-\bar{\mu}) A+(k-\mu) \bar{A}
$$

by (4) and (5). From this, (3), (41), and (32), it follows that

$$
\begin{aligned}
\boldsymbol{A} \boldsymbol{A}_{a, b}= & (a+1) \boldsymbol{A}_{a+1, b}+(a+1)(\bar{k}-\bar{\mu}) \boldsymbol{A}_{a+1, b-1}+(a \lambda+b(k-\mu)) \boldsymbol{A}_{a, b} \\
& +(b+1) \mu \boldsymbol{A}_{a-1, b+1}+(n-a-b+1) k \boldsymbol{A}_{a-1, b} \\
\overline{\boldsymbol{A}} \boldsymbol{A}_{a, b}= & (b+1) \boldsymbol{A}_{a, b+1}+(a+1) \bar{\mu} \boldsymbol{A}_{a+1, b-1}+(a(\bar{k}-\bar{\mu})+b \bar{\lambda}) \boldsymbol{A}_{a, b} \\
& +(b+1)(k-\mu) \boldsymbol{A}_{a-1, b+1}+(n-a-b+1) \bar{k} \boldsymbol{A}_{a, b-1} .
\end{aligned}
$$

Observe that the above identities correspond to the expansions of

$$
\theta_{j, h} \cdot k_{a, b} \mathcal{K}_{a, b}(j, h), \quad \bar{\theta}_{j, h} \cdot k_{a, b} \mathcal{K}_{a, b}(j, h),
$$

respectively, in terms of the polynomials $k_{c, d} \mathcal{K}_{c, d}\left((c, d) \in \Lambda_{n}\right)$. See also [17, Section 6]. By (35), we now rewrite these as recurrence relations for the $\hat{\mathcal{K}}_{a, b}=\sqrt{k_{a, b}} \mathcal{K}_{a, b}$ and then let $n \rightarrow \infty$. For example, the coefficient of $\hat{\mathcal{K}}_{a, b}$ in $x \hat{\mathcal{K}}_{a, b}$ is given by

$$
\frac{a \lambda+b(k-\mu)}{\sqrt{n k}}=\frac{a(r+s+k+r s)-b r s}{k} \sqrt{\frac{k}{n}},
$$

where we have used (15). We claim that this coefficient converges to

To see this, we note that

$$
(a \kappa+b \bar{\kappa}) \frac{\sqrt{\kappa}}{\omega} .
$$

$$
-r s(f+g)=k v-k^{2}+(r+s) k
$$

by virtue of (9) and (10), from which it follows that (cf. (7))

$$
-\frac{r s}{k} \rightarrow \frac{\bar{\kappa}}{\omega} \text {. }
$$

If $\kappa>0$ then the claim follows directly from (47). On the other hand, if $\kappa=0$ then the coefficient (46) converges to zero since the first factor of the RHS is bounded 
by (6) and (47), and hence the claim again holds. The other coefficients can be computed similarly and more easily using (15), (33), and (47) (and the complement versions of (15) and (47)). It follows that

$$
\begin{aligned}
x \mathcal{C H}_{a, b}= & \sqrt{a+1} \mathcal{C H} \mathcal{H}_{a+1, b}+\sqrt{(a+1) b} \frac{\kappa \sqrt{\kappa}}{\omega} \mathcal{C H}_{a+1, b-1} \\
& +(a \kappa+b \bar{\kappa}) \frac{\sqrt{\kappa}}{\omega} \mathcal{C H} \mathcal{H}_{a, b}+\sqrt{a(b+1)} \frac{\kappa \sqrt{\kappa}}{\omega} \mathcal{C H}_{a-1, b+1}+\sqrt{a} \mathcal{C H}_{a-1, b}, \\
y \mathcal{C H}_{a, b}= & \sqrt{b+1} \mathcal{C H}{ }_{a, b+1}+\sqrt{(a+1) b} \frac{\bar{\kappa} \sqrt{\kappa}}{\omega} \mathcal{C H} \mathcal{H}_{a+1, b-1} \\
& +(a \kappa+b \bar{\kappa}) \frac{\sqrt{\bar{\kappa}}}{\omega} \mathcal{C H}_{a, b}+\sqrt{a(b+1)} \frac{\bar{\kappa} \sqrt{\kappa}}{\omega} \mathcal{C H}_{a-1, b+1}+\sqrt{b} \mathcal{C H}_{a, b-1}
\end{aligned}
$$

for $a, b=0,1,2, \ldots$ In particular, it follows inductively that the $\mathcal{C H}_{a, b}$ form a linear basis of $\mathbb{C}[x, y]$. More precisely, the coefficients of every monomial $x^{c} y^{d}$ in terms of the $\mathcal{C H}_{a, b}$ are the limits of those of $x^{c} y^{d}$ in terms of the $\hat{\mathcal{K}}_{a, b}$.

We are now ready to establish the orthogonality relation for the $\mathcal{C} \mathcal{H}_{a, b}$. Let $\nu$ be the probability measure in Theorem [2.1(iii), and recall that $\nu_{G} \square_{n}, \bar{G}^{\square n}$ converges weakly to $\nu$. Pick any monomial $x^{c} y^{d}$. Observe that

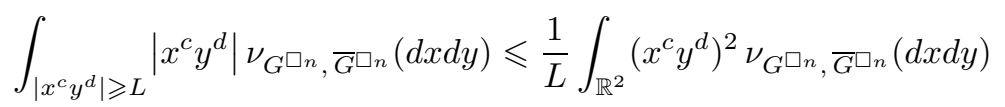

for every $L>0$, and that the integral in the RHS is uniformly bounded with respect to $n$ by virtue of (38) and the above comment. Hence we have

$$
\lim _{L \rightarrow \infty} \sup _{n} \int_{\left|x^{c} y^{d}\right| \geqslant L}\left|x^{c} y^{d}\right| \nu_{G}^{\square n}, \bar{G} \square n(d x d y)=0 .
$$

It is well known (see e.g., [1, Lemma 8.4.3]) that this implies that

$$
\int_{\mathbb{R}^{2}} x^{c} y^{d} \nu_{G}^{\square n}, \bar{G} \square n(d x d y) \rightarrow \int_{\mathbb{R}^{2}} x^{c} y^{d} \nu(d x d y) .
$$

Combining this with (38), it follows that

$$
\int_{\mathbb{R}^{2}} \mathcal{C H}_{a, b}(x, y) \mathcal{C H}_{c, d}(x, y) \nu(d x d y)=\delta_{a, c} \delta_{b, d}
$$

for $a, b, c, d=0,1,2, \ldots$.

Remark 6.1. The discussions in this section work for the other cases in Theorem 2.1 as well, and we obtain (special cases of) the bivariate Charlier polynomials studied by Genest, Miki, Vinet, and Zhedanov [7 for Theorem 2.1(i) and (ii), and the products of univariate Hermite polynomials for Theorem 2.1(iv); cf. 6, Section 5.1.3]. We omit the details. Genest et al. [7, Section 10] also showed among other results that the bivariate Charlier polynomials can be obtained from the bivariate Krawtchouk polynomials by a limit process. It should be remarked that, unless $\kappa=0$ or $\bar{\kappa}=0$, the bivariate Charlier-Hermite polynomials $\mathcal{C H}_{a, b}$ differ from affine transformations of the products of univariate Poisson and Hermite polynomials, which form another system of orthogonal polynomials with respect to the measure $\nu$ in Theorem 2.1(iii); cf. (45).

Remark 6.2. The above proof of the orthogonality relation (48) for the $\mathcal{C H}_{a, b}$ is based on the weak convergence $\nu_{G} \square n, \bar{G}^{\square} n$ 
that (48) is valid for all the parameters $\kappa, \bar{\kappa} \geqslant 0$ with $\omega=\kappa+\bar{\kappa}>0$. On the other hand, the bivariate Krawtchouk polynomials $\mathcal{K}_{a, b}$ and their orthogonality relation (cf. (38)) have been discussed at a purely algebraic/parametric level [17, 18, and it seems that we may also establish (48) in full generality by taking limits at this level and therefore without any reference to specific constructions of strongly regular graphs as in Example 5.4.

\section{ACKNOWLEDGMENTS}

This work, except Section 6, is part of the Ph.D. thesis of JVSM [19]. NO was supported by JSPS KAKENHI Grant Number JP16H03939. HT was supported by JSPS KAKENHI Grant Numbers JP25400034 and JP17K05156.

\section{REFERENCES}

[1] V. I. Bogachev, Measure theory, Vol. II, Springer-Verlag, Berlin, 2007.

[2] A. E. Brouwer, SRG family parameters, http://www.win.tue.nl/ aeb/graphs/srghub.html.

[3] A. E. Brouwer and W. H. Haemers, Spectra of graphs, Springer, New York, 2012.

[4] E. R. van Dam, J. H. Koolen, and H. Tanaka, Distance-regular graphs, Electron. J. Combin. (2016) \#DS22; arXiv 1410.6294

[5] P. Delsarte, An algebraic approach to the association schemes of coding theory, Philips Res. Rep. Suppl. No. 10 (1973).

[6] C. F. Dunkl and Y. Xu, Orthogonal polynomials of several variables (2nd edn.), Cambridge University Press, Cambridge, 2014.

[7] V. X. Genest, H. Miki, L. Vinet, and A. Zhedanov, The multivariate Charlier polynomials as matrix elements of the Euclidean group representation on oscillator states, J. Phys. A 47 (2014) 215204; arXiv:1401.7901

[8] C. D. Godsil, Generalized Hamming schemes, manuscript (2010); arXiv:1011.1044

[9] R. C. Griffiths, Orthogonal polynomials on the multinomial distribution, Austral. J. Statist. 13 (1971) 27-35.

[10] F. A. Grünbaum, The Rahman polynomials are bispectral, SIGMA Symmetry Integrability Geom. Methods Appl. 3 (2007) 065; arXiv:0705.0468.

[11] Y. Hashimoto, A. Hora, and N. Obata, Central limit theorems for large graphs: method of quantum decomposition, J. Math. Phys. 44 (2003) 71-88.

[12] Y. Hashimoto, N. Obata, and N. Tabei, A quantum aspect of asymptotic spectral analysis of large Hamming graphs, in: T. Hida and K. Saitô (eds.), Quantum information III, World Scientific Publishing, River Edge, NJ, 2001, pp. 45-57.

[13] M. R. Hoare and M. Rahman, A probabilistic origin for a new class of bivariate polynomials, SIGMA Symmetry Integrability Geom. Methods Appl. 4 (2008) 089; arXiv:0812.3879

[14] A. Hora, Central limit theorems and asymptotic spectral analysis on large graphs, Infin. Dimens. Anal. Quantum Probab. Relat. Top. 1 (1998) 221-246.

[15] A. Hora and N. Obata, Quantum probability and spectral analysis of graphs, Springer, Berlin, 2007.

[16] A. Hora and N. Obata, Asymptotic spectral analysis of growing regular graphs, Trans. Amer. Math. Soc. 360 (2008) 899-923.

[17] P. Iliev and P. Terwilliger, The Rahman polynomials and the Lie algebra $\mathfrak{s l}_{3}(\mathbb{C})$, Trans. Amer. Math. Soc. 364 (2012) 4225-4238; arXiv:1006.5062.

[18] H. Mizukawa and H. Tanaka, $(n+1, m+1)$-hypergeometric functions associated to character algebras, Proc. Amer. Math. Soc. 132 (2004) 2613-2618.

[19] J. V. S. Morales, On extensions of commutative association schemes, thesis, Tohoku University, 2017.

[20] A. Neumaier, Strongly regular graphs with smallest eigenvalue $-m$, Arch. Math. (Basel) 33 $(1979 / 80) 392-400$

[21] H. Tarnanen, On extensions of association schemes, in: H. Laakso and A. Salomaa (eds.), The very knowledge of coding, University of Turku, Institute for Applied Mathematics, Turku, 1987, pp. $128-142$. 
[22] P. Terwilliger, The subconstituent algebra of an association scheme I, J. Algebraic Combin. 1 (1992) 363-388.

Mathematics and Statistics Department, College of Science, De la Salle University, Manila, Philippines

E-mail address: john.vincent.morales@dlsu.edu.ph

Research Center for Pure and Applied Mathematics Graduate School of Information Sciences, Tohoku University, Sendai, Japan

E-mail address: obata@math.is.tohoku.ac.jp

URL: http://www .math.is.tohoku.ac.jp/ obata/

Research Center for Pure and Applied Mathematics, Graduate School of InformaTion Sciences, Tohoku University, Sendai, Japan

E-mail address: htanaka@tohoku.ac.jp

$U R L:$ http://www.math.is.tohoku.ac.jp/ ${ }^{\sim}$ htanaka/ 